\title{
Susceptibility of Pediococcus isolates to antimicrobial compounds in relation to hop-resistance and beer-spoilage Monique Haakensen ${ }^{1}$, David M Vickers² ${ }^{2}$ and Barry Ziola*1
}

\author{
Address: ${ }^{1}$ Department of Pathology and Laboratory Medicine, Room 2841 Royal University Hospital, 103 Hospital Drive, University of \\ Saskatchewan, Saskatoon, SK S7N 0W8, Canada and '2Department of Computer Science, 110 Science Place, University of Saskatchewan, \\ Saskatoon, SK, S7N 5C9, Canada \\ Email: Monique Haakensen - m.haakensen@usask.ca; David M Vickers - david.vickers@usask.ca; Barry Ziola* - b.ziola@usask.ca \\ * Corresponding author
}

Published: 7 September 2009

BMC Microbiology 2009, 9:190 doi:10.1 186/147/-2180-9-190
Received: 30 January 2009

Accepted: 7 September 2009

This article is available from: http://www.biomedcentral.com/I47|-2/80/9/190

(C) 2009 Haakensen et al; licensee BioMed Central Ltd.

This is an Open Access article distributed under the terms of the Creative Commons Attribution License (http://creativecommons.org/licenses/by/2.0), which permits unrestricted use, distribution, and reproduction in any medium, provided the original work is properly cited.

\begin{abstract}
Background: Though important in the context of food microbiology and as potential pathogens in immuno-compromised humans, bacterial isolates belonging to the genus Pediococcus are best known for their association with contamination of ethanol fermentation processes (beer, wine, or fuel ethanol). Use of antimicrobial compounds (e.g., hop-compounds, Penicillin) by some industries to combat Pediococcus contaminants is long-standing, yet knowledge about the resistance of pediococci to antimicrobial agents is minimal. Here we examined Pediococcus isolates to determine whether antibiotic resistance is associated with resistance to hops, presence of genes known to correlate with beer spoilage, or with ability to grow in beer.
\end{abstract}

Results: Lactic acid bacteria susceptibility test broth medium (LSM) used in combination with commercially available GPN3F antimicrobial susceptibility plates was an effective method for assessing antimicrobial susceptibility of Pediococcus isolates. We report the finding of Vancomycinsusceptible Pediococcus isolates from four species. Interestingly, we found that hop-resistant, beerspoilage, and beer-spoilage gene-harbouring isolates had a tendency to be more susceptible, rather than more resistant, to antimicrobial compounds.

Conclusion: Our findings indicate that the mechanisms involved in conferring hop-resistance or ability to spoil beer by Pediococcus isolates are not associated with resistance to antibiotics commonly used for treatment of human infections. Also, Vancomycin-resistance was found to be isolate-specific and not intrinsic to the genus as previously believed.

\section{Background}

Isolates from the genus Pediococcus are particularly problematic for the brewing industry where hop-compounds are used to provide flavour to beer. Hop-compounds are antimicrobial in that they dissipate the trans-membrane $\mathrm{pH}$ gradient of microbes, thereby inhibiting growth and potential spoilage of product [1]. As pediococci are also used as beneficial microbes in the context of food micro- biology and animal husbandry (e.g., wine, cheese, and yogurt industries as well as for the production of silage), the emergence of hop-resistant Pediococcus isolates in the brewing industry is of broader interest. These isolates frequently harbour one or more ATP-binding cassette type multidrug resistance (ABC MDR) genes, suggesting that resistance to hop-compounds may also confer resistance to other antimicrobial compounds [2]. We have previ- 
ously shown that several genes can be correlated with ability of Pediococcus isolates to grow in beer and to resist the antimicrobial activity of hop-compounds [3-5]. These are the ABC MDR genes $A B C 2, b s r A, b s r B,[6]$ and hor $A$ [2], a putative divalent cation transporter known as hitA [7], and horC which codes for a protein possessing little homology to any known protein $[8,9]$.

Because, many pediococci possess special growth requirements, conventional antimicrobial-sensitivity testing media have been demonstrated to be unsuitable for testing of Pediococcus isolates for antimicrobial resistance [1012]. However, enriched media that permits growth of pediococci may inhibit the antimicrobial activity of some compounds under investigation. Previously, antimicrobial susceptibility testing of Pediococcus isolates has been attempted by several methods, many of which are performed using some variety of agar diffusion $[10,11,13,14]$. However, more recently, dilution methods have been preferred over diffusion tests as the former allow for determination of minimum inhibitory concentrations (MICs), which is a more reliable and reproducible indicator of resistance $[10,11]$. For these reasons, lactic acid bacteria susceptibility test broth medium (LSM), which was recently developed by Klare et al. [11], should be considered the new testing standard for assessing the antimicrobial resistance spectra of lactic acid bacteria. Despite this medium being shown to be very effective for establishing antimicrobial susceptibilities of two species of Pediococcus, namely, P. acidilactici, and P. pentosaceus [10], it previously has not been used to study the prevalence, and spectrum, of antimicrobial resistance among other members of the genus.

Overall, the use of antimicrobial compounds by industries such as animal husbandry, brewing, and fuel ethanol to combat Pediococcus contaminants (e.g., hop-compounds, Penicillin, and Virginiamycin which is structurally similar to Synercid) is long-standing. However, knowledge about the resistance of pediococci to antimicrobial agents is minimal [12]. As such, the focus of this research was to determine whether the use of antimicrobial hop-compounds in the brewing industry is associated with an increase in the overall antimicrobial resistance of Pediococcus isolates. Here we report on the testing of isolates from six species of the genus Pediococcus against 17 antimicrobial compounds using LSM broth in commercially available Sensititre GPN3F Gram-positive MIC plates (TREK Diagnostic Systems, Cleveland OH).

\section{Results}

\section{Antimicrobial susceptibility testing}

Twenty-nine isolates, including six species of the Pediococcus genus were tested. Distribution of isolates by species and their ability to grow in beer is given in Table 1. Antimicrobial resistance testing was reproducible and the LSM by itself (containing no antimicrobial compounds) was permissive to the rapid growth of all Pediococcus isolates tested. All isolates used in this study were capable of producing visible turbidity in LSM broth after an incubation period of 24 hours. Isolates were cultured for a period of 48 hours in GPN3F plates so as to allow formation of larger bacterial pellets and thus a more accurate determination of the MIC for a given antibiotic. All control wells in the GPN3F plates produced appropriate results. Eight of the 29 isolates were randomly selected and tested in duplicate by the same method, and no variance in MICs was observed. The antimicrobial compounds and dilutions tested by the GPN3F antimicrobial susceptibility plates are listed in Additional file 1.

Table I: Pediococcus isolates.

\begin{tabular}{|c|c|c|c|c|c|c|c|}
\hline \multirow{2}{*}{\multicolumn{2}{|c|}{ Species }} & \multirow[t]{2}{*}{$\mathbf{N}$} & \multicolumn{3}{|c|}{ Origin } & \multicolumn{2}{|c|}{ Growth in Beer ${ }^{a}$} \\
\hline & & & Brewery & Otherb $^{b}$ & Unknown & + & - \\
\hline acidilactici & & 6 & 4 & I & 1 & 1 & 5 \\
\hline claussenii & & 12 & 12 & 0 & 0 & 11 & 1 \\
\hline & ropyc & (5) & $(5)$ & $(0)$ & $(0)$ & (5) & (0) \\
\hline & non-ropyd & (7) & (7) & (0) & (0) & (6) & (I) \\
\hline damnosus & & I & 1 & 0 & 0 & 0 & I \\
\hline inopinatus & & 1 & 1 & 0 & 0 & 0 & I \\
\hline parvulus & & 5 & 0 & 5 & 0 & 1 & 4 \\
\hline & ropy & (I) & $(0)$ & (I) & $(0)$ & $(0)$ & ( 1 ) \\
\hline & non-ropy & (4) & (0) & (4) & (0) & (I) & (3) \\
\hline pentosaceus & & 4 & i & 2 & i & 0 & 4 \\
\hline Total & & 29 & 19 & 8 & 2 & 13 & 16 \\
\hline
\end{tabular}

a Previously reported by Haakensen et al. [3,4].

b Isolates of known non-brewery origin, specific origins are provided in Additional file 2.

c, d Isolates positive and negative for exopolysaccharide rope production, respectively. 


\section{Distribution of MIC by species, isolate, and ropy phenotype}

Resistance to the 17 antimicrobial compounds and hopcompounds was determined, and the antimicrobial compounds to which resistant isolates of Pediococcus were found are given in Additional file 1. For the majority of the 29 isolates tested, a moderate degree of susceptibility was shown to each of the antibiotics and a MIC value could be determined. However, for two of the antibiotics (i.e., Vancomycin and Ciprofloxacin), the majority of isolates (72\% and 52\%, respectively) grew in the presence of the antibiotic at all concentrations tested. Additionally, $48 \%$ of isolates were hop-resistant. When Pediococcus claussenii and Pediococcus parvulus were assessed on the basis of ropy (i.e., exopolysaccharide-producing) phenotype, there was no significant difference found among the MICs for each antibiotic [Additional files 1 and 2].

Analysis of antimicrobial resistance according to Pediococcus species demonstrated that just over half of the antibiotics (9/17) had significantly different MICs for different species (Table 2 and Additional files 1 and 2). The nonparametric Kruskal-Wallis $H$-test was used to test for equality in population medians. This test is an extension of the Mann-Whitney $U$-test which is designed to examine whether two samples of observations come from the same distribution. Unfortunately, post-hoc analyses to determine which of the six species had significantly different MICs for each antibiotic was not possible due to the low number of isolates per species. However, when P. claussenii isolates were compared to isolates of the other species combined, P. claussenii had significantly lower MICs (Mann-Whitney $U$-test, $p<0.05$ ) for all antimicrobial compounds tested, except for Erythromycin, Clindamycin, Daptomycin, and Vancomycin (data not shown).

Table 2: Antimicrobial compounds having significantly different MICs among the six Pediococcus species.

\begin{tabular}{ll}
\hline Antimicrobial compound & $\boldsymbol{p}^{\text {-value }} \mathbf{a}^{\mathbf{a}}$ \\
\hline Ampicillin & $<0.02$ \\
Ceftriaxone & $<0.02$ \\
Ciprofloxacin & $<0.02$ \\
Daptomycin & $<0.02$ \\
Gatifloxacin & $<0.01$ \\
Gentamicin & $<0.05$ \\
Levofloxacin & $<0.01$ \\
Penicillin & $<0.02$ \\
Synercid & $<0.05$
\end{tabular}

a $p$-value corresponds to the $H$-test statistic as derived from the nonparametric Kruskal-Wallis $H$-test which tests for equality in population medians where there are three or more groups.

\section{Distribution of MIC by presence of genes associated with} beer-spoilage andlor hop-resistance

Whether any of the beer-spoilage and/or hop resistancecorrelated genes $A B C 2$, bsr $A$, bs $B$, hit $A$, hor $A$, and hor $C$ were associated with any of the antimicrobial MICs was determined [Additional file 2]. Of these six genes, hitA, hor $C$, and $A B C 2$, did not occur with sufficient frequency to be analyzed statistically. The $b s r A, b s r B$, and horA genes unexpectedly demonstrated significant associations not with antibiotic resistance, but with susceptibility to antimicrobial compounds ( $b s r A$ and $b s r B$ with Ampicillin, Levofloxacin, Penicillin, Ciprofloxacin, Gatifloxacin, and Oxacillin + 2\% NaCl; horA with Erythromycin).

\section{Distribution of MIC by hop-resistance phenotype}

Fourteen of the 29 isolates (48.3\%) were deemed resistant to hop-compounds as tested by the hop-gradient agar plate with ethanol method. When the isolates categorized according to susceptibility or resistance to hop-compounds had their MICs compared using the Mann-Whitney $U$-test, $29.4 \%$ (5/17) of the antimicrobial compounds had significantly lower MICs for the hop-resistant isolates (Table 3). Of these five antimicrobials, only Ciprofloxacin showed a significant correlation with hop-resistance. Unexpectedly, the correlation was a negative one (Spearman's $\rho=-0.47, p<0.01)$, since as the MIC for Ciprofloxacin increased, the probability of an isolate's growth in the presence of hop-compounds decreased.

\section{Distribution of MIC by ability to grow in beer}

Of the 29 Pediococcus isolates tested, 13 (44.8\%) were capable of growing in beer. The results of testing for an association between antibiotic susceptibility and growth in beer are given in Table 4 . Based on a Mann-Whitney $U$ test, eight of the 17 antibiotics tested demonstrated a significantly lower MIC in those isolates that could grow in beer.

\section{Discussion}

The finding of Pediococcus isolates that showed only moderate resistance to Vancomycin is discordant with other studies to date which have consistently reported the genus Pediococcus to be intrinsically Vancomycin-resistant $[10,12-14]$. The isolates that were not resistant to all concentrations of Vancomycin tested were from the species $P$. acidilactici $(\mathrm{N}=1), P$. claussenii (Ropy, $\mathrm{N}=1$; Non-ropy, $\mathrm{N}$ =3), P. damnosus $(\mathrm{N}=1)$, and $P$. parvulus (Non-ropy, $\mathrm{N}=$ 2 ), suggesting that the phenomenon is not the product of a clonal event. It has previously been shown that intrinsic Vancomycin resistance in $P$. pentosaceus is due to a modified peptidoglycan precursor ending in D-Ala-D-lactate [15]. While this may also be the mechanism used by other Vancomycin-resistant pediococci, it is likely that the eight susceptible isolates do not possess this mechanism. Because media previously used for Pediococcus antimicro- 
Table 3: Antimicrobial compounds having significantly lower MICs in hop-resistant isolates ${ }^{\mathbf{a}}$.

\begin{tabular}{|c|c|c|c|}
\hline \multirow[t]{2}{*}{ Antimicrobial compound } & \multicolumn{2}{|c|}{ Median and Distribution of MIC $(\mu \mathrm{g} / \mathrm{ml})$} & \multirow[t]{2}{*}{ p-value } \\
\hline & Hop-resistant & Hop-sensitive & \\
\hline Ampicillin & $0.25(0.12-4)$ & $\mathrm{I}(0.12-4)$ & $<0.05$ \\
\hline Ciprofloxacin & $2(0.5-N R c)$ & $4(0.5-N R)$ & $<0.05$ \\
\hline Gatifloxacin & I (0.5-8) & $4(\mathrm{I}-\mathrm{NR})$ & $<0.05$ \\
\hline Penicillin & $0.12(0.06-N R)$ & $2(0.06-N R)$ & $<0.02$ \\
\hline Rifampin & $0.5(0.5-2)$ & I (0.5-NR) & $<0.05$ \\
\hline
\end{tabular}

a Hop-resistance is as determined by the hop-gradient agar plate with ethanol method.

${ }^{\mathrm{b}} \mathrm{p}$-value corresponds to $U$-test statistic as derived from the non-parametric Mann-Whitney $U$-test which is designed to examine whether two samples of observations came from the same distribution.

cNR; MIC not reached, isolate could grow at highest concentration of antibiotic tested.

bial susceptibility testing have since been shown to be inappropriate for such testing (11), it is possible that the earlier finding of intrinsic Pediococcus Vancomycin-resistance was an artifact of the testing medium used, rather than reflective of pediococci genetic content.

The ropy phenotype did not associate with resistance to any of the antimicrobial compounds tested. This was an unexpected result as the ropy phenotype acts to create a biofilm which is expected to act as a physical barrier for the bacteria, putatively protecting them from the antimicrobial compounds. Why no associations were found is unclear. It may be that the type of exopolysaccharide matrix produced by these isolates did not result in a sufficiently dense matrix so as to inhibit the passage of antimicrobial compounds. Alternatively, the amount of energy expended on the production of exopolysaccharide may have caused a decreased ability to grow in the presence of the antimicrobial compounds, despite the partial antimicrobial barrier created by the exopolysaccharide.

Of particular interest to the brewing industry is the presence in pediococci of hop-resistance or beer-spoilage cor- related genes $(A B C 2, b s r A, b s r B$, hitA, hor $A$, and hor $C)$. Of these six genes, only hor $A$ has been conclusively shown to function as a multidrug transporter, however, the $A B C 2$, $b s r A$, and $b s r B$ genes are highly similar to known $A B C$ MDR genes, and the hit gene is similar to divalent cation transporters. As such, all six of these beer-spoilage or hopresistance correlated genes were assessed for associations with antimicrobial resistance. The genes hitA, hor $C$, and $A B C 2$ did not occur with sufficient frequency to determine statistical correlation [Additional file 2]. It is important to note that, as was found for ability to grow in beer, the $b s r A, b s r B$, and hor $A$ genes did not demonstrate significant associations with resistance to any of the antibiotics tested, but rather with susceptibility.

When MIC was compared to ability of isolates to grow in beer, eight of the 17 antibiotics that we tested surprisingly demonstrated a significantly lower MIC in isolates that could grow in beer. The eight antibiotics included Synercid, Ampicillin, Levofloxacin, Penicillin, Ciprofloxacin, Sulfamethoxazole/Trimethoprim, Gatifloxacin, and Oxacillin $+2 \% \mathrm{NaCl}$. This suggests that, despite repeated exposure to antimicrobial hop-compounds in the brewery

Table 4: Antimicrobial compounds having significantly lower MICs in isolates able to grow in beer.

\begin{tabular}{|c|c|c|c|}
\hline \multirow[t]{2}{*}{ Antimicrobial compound } & \multicolumn{2}{|c|}{ Median and Distribution of MIC $(\mu \mathrm{g} / \mathrm{ml})$} & \multirow[t]{2}{*}{ p-value ${ }^{a}$} \\
\hline & Grow in Beer & Cannot grow in beer & \\
\hline Ampicillin & $0.25(0.12-4)$ & $2(0.12-4)$ & $<0.01$ \\
\hline Ciprofloxacin & $2\left(0.5-N^{b}\right)$ & $4(0.5-N R)$ & $<0.01$ \\
\hline Gatifloxacin & I (0.25-8) & 4 (I-NR) & $<0.01$ \\
\hline Levofloxacin & 2 (0.5-NR) & $16(I-N R)$ & $<0.05$ \\
\hline Oxacillin $+2 \% \mathrm{NaCl}$ & $0.25(0.25-4)$ & I (0.25-NR) & $<0.02$ \\
\hline Penicillin & $0.12(0.12-N R)$ & I (0.06-NR) & $<0.01$ \\
\hline Synercid & $0.5(0.12-1)$ & $\mathrm{I}(0.25-2)$ & $<0.05$ \\
\hline Trimethoprim/Sulfamethoxazole & $0.5 / 9.5(0.5 / 9.5-\mathrm{NR})$ & $\mathrm{R}(0.5 / 9.5-\mathrm{NR})$ & $<0.05$ \\
\hline
\end{tabular}

ap-value corresponds to $U$-test statistic as derived from the non-parametric Mann-Whitney $U$-test which is designed to examine whether two samples of observations came from the same distribution.

${ }^{b}$ NR; MIC not reached, isolate could grow at highest concentration of antibiotic tested. 
setting, Pediococcus isolates capable of growing in the beer tend to be more susceptible to commonly used antimicrobial compounds than are isolates which cannot grow in beer. It is possible that this association may actually be independent of the presence of hop-compounds, instead being an indication of the environment encountered within the brewery environment by the beer-spoilage isolates. Although beer-spoilage bacteria must originate from outside the brewery, isolates capable of growing in beer have presumably become highly acclimatized or especially adapted to grow in the beer environment. Ideally, beer will not contain any wild yeasts or bacteria and, as such, contaminating pediococci are growing in an environment that does not contain a plethora of antimicrobial compounds naturally created by other organisms living in the same environment. Based on this scenario, Pediococcus isolates entering the brewery environment from outside sources (e.g., plant materials such as hop cones or barley) would possess mechanisms of resistance against multiple antimicrobial compounds. However, upon entering the brewery environment which should be free of other competing microbes, the pediococci would encounter no selective pressures other than hop-compounds and thus fail to maintain the genetic mechanisms for antimicrobial resistance.

It is curious to note that the $b s r A$ and $b s r B$ genes, hopresistance, and beer-spoilage are all significantly negatively-associated with resistance to Ciprofloxacin. Moreover, although horA is strongly correlated to ability to grow in beer, this gene does not show any association (negative or otherwise) with Ciprofloxacin resistance. While the underlying mechanism for this association with lowered resistance to Ciprofloxacin is unknown, it strongly suggests that hop-resistance, and in turn beer-spoilage, is linked to the presence of the $b s r A$ and $b s r B$ genes, while the horA gene may simply be correlated by chance to ability of Pediococcus isolates to spoil beer. That is to say, because the $b s r A$ and $b s r B$ genes (like the beer-spoilage phenotype) are negatively correlated to ciprofloxacin resistance, while the horA gene is not, the $b s r A$ and $b s r B$ genes are likely more closely associated with beer-spoilage than is the horA gene.

\section{Conclusion}

Testing the susceptibility of Pediococcus isolates to antimicrobial compounds was effective using LSM in GPN3F antimicrobial susceptibility testing plates. In contrast with previous studies, we found Pediococcus isolates that are not intrinsically resistant to Vancomycin. A significant negative association was identified between resistance to Ciprofloxacin and the presence of the $b s r A$ and $b s r B$ genes as well as the hop-resistant and beer-spoilage phenotypes. Significantly lower MICs to antimicrobial compounds were found in isolates that were hop-resistant and/or capable of growing in beer. Similarly, the presence of genes previously correlated with beer-spoilage (i.e., bsrA, $b s r B$, and horA) was also found to be associated with significantly lower MICs to several of the antimicrobial compounds tested. These results suggest that the ongoing use of the antimicrobial hop-compounds in the brewing industry and the phenomenon of hop-resistance mediated by ATP-binding cassette type multi-drug transporters is not associated with the emergence of greater antimicrobial resistance in beer-spoilage pediococci.

\section{Methods}

\section{Bacterial growth in beer}

A list of the bacterial species tested is provided in Table 1, with the isolates comprising 29 pediococci (six species) and including six ropy (exopolysaccharide producing) strains. Speciation of bacterial strains was determined (or in the case of culture collection strains, confirmed) by sequencing of the first three variable regions of the $16 \mathrm{~S}$ rRNA gene as previously described [4]. Parameters for induction of bacteria to grow in beer were as described by Haakensen et al. [4]. In brief, assessment of bacterial isolate growth in beer required adaptation of the bacteria using modified mMRS broth (MRS medium with Tween $80^{\mathrm{TM}}$ omitted [4]) supplemented with incremental concentrations of beer. Beer 1 was a filter-sterilized $4 \% \mathrm{v} / \mathrm{v}$ alcohol beer, pH 4.2 and averaging 9.8 bitterness units, while Beer 2 was a pasteurized 5\% v/v alcohol beer, $\mathrm{pH} 3.8$ and averaging 11 bitterness units. Bacteria capable of growing in either beer were considered to be beer-spoilers. Prior to testing for hop-resistance as described in Sections 2.2 and 2.3 , bacteria were initially grown in $50 \% 2 \times$ mMRS and $50 \%$ Beer 2 as described by Haakensen et al. [4]. Bacteria were then grown at $30^{\circ} \mathrm{C}$ for $16-24$ hours in $15 \% 2 \times$ mMRS and $85 \%$ Beer 2 .

\section{Ability of bacteria to resist hop-compounds}

All bacterial isolates were tested for resistance to hopcompounds by the hop-gradient mMRS agar plate containing ethanol method as described by Haakensen $e t$ al. [5]. The ability of each isolate to grow on the hop-gradient mMRS agar plate containing ethanol is provided in Additional file 2 .

\section{Presence of beer-spoilage related genes}

All bacterial isolates were tested for the presence of the putative beer-spoilage associated genes $A B C 2, b s r A, b s r B$, hit $A$, hor $A$, and hor $C$ as previously described by Haakensen et al. $[3,4,6]$. The presence or absence of these genes in each isolate is recorded in Additional file 2. Only $b s r A$, $b s r B$, and horA occurred with sufficient frequency for use in subsequent statistical analyses. 


\section{Antimicrobial susceptibility testing}

Antimicrobial susceptibility testing was performed using LSM and Sensititre GPN3F Gram-positive MIC plate (TREK Diagnostic Systems, Cleveland $\mathrm{OH}$ ). Additional file 1 provides a list of antimicrobial compounds and concentration ranges tested. The GPN3F plates contained vacuum-dried antimicrobial compounds which were rehydrated when LSM containing the bacterial inoculate was added. Bacteria were diluted to approximately $10^{3}$ $10^{4} \mathrm{cfu} / \mathrm{ml}$ in LSM (confirmed by colony counting on MRS agar plates) and $100 \mu$ were inoculated into each well of a Sensititre GPN3F plate. Bacteria were grown for 48 hours in a candle jar at $30^{\circ} \mathrm{C}$. The MICs $(\mu \mathrm{g} / \mathrm{ml})$ were determined based on appearance of visible bacterial pellets in the bottom of wells.

\section{Statistical analysis}

Non-parametric Mann-Whitney $U$ (when testing for a difference between 2 independent samples) or Kruskal-Wallis $H$ (in the case of $>2$ independent samples) tests were used to compare the MICs for the 17 antibiotics to determine whether antibiotic resistance had an association with resistance to hops, presence of known genes associated with hop-resistance, antibiotic-resistance, as well as with the ability of Pediococcus isolates to grow in beer.

For some of the analyses, the indicator (categorical) variable of resistance or susceptibility to hop-compounds was created as described by Haakensen et al. [5]. Specifically, if a Pediococcus isolate was observed to have positive growth $(>3 \mathrm{~cm})$ on hop-gradient agar with ethanol plates, then that isolate was categorized as 'hop-resistant'. For this indicator variable, Fisher's exact test and Spearman's correlation coefficient $\rho$ were used for the comparison of gene presence and antibiotic resistance, respectively, with the hop-resistance indicator variable. All tests of significance were performed at $\alpha=0.05$ using SPSS Statistical Software for Windows (SPSS Inc., Chicago, IL, version 14.0).

\section{Authors' contributions}

$\mathrm{MH}$ conceived the study, participated in the design, performed laboratory work, and drafted parts of the manuscript. DMV performed statistical analysis and drafted parts of the manuscript. BZ conceived the study, participated in its design and coordination, edited the manuscript, and is the holder of the research grand used to fund the study. All authors have read and approved the final manuscript.

\section{Additional material}

\section{Additional file 1}

Range of minimum inhibitory concentrations of antimicrobial compounds summarized by species. The data provided indicate the range of concentrations tested for each antibiotic and the range of MICs obtained for each Pediococcus species.

Click here for file

[http://www.biomedcentral.com/content/supplementary/14712180-9-190-S1.docx]

\section{Additional file 2}

Isolate and antibiotic MIC information. Information regarding the isolates used in the study, and the MICs obtained for each antibiotic by each isolate.

Click here for file

[http://www.biomedcentral.com/content/supplementary/1471-

2180-9-190-S2.xls]

\section{Acknowledgements}

M.H. was awarded the Coors Brewing Company, Cargill Malt, and Miller Brewing Company Scholarships from the American Society of Brewing Chemists Foundation, and was the recipient of Graduate Scholarships from the College of Medicine, University of Saskatchewan. D.M.V. currently holds a Regional Partnership Program Doctoral Research Award from the Canadian Institutes of Health Research. This research was supported by the Natural Science and Engineering Research Council of Canada through Discovery Grant 24067-05.

\section{References}

I. Simpson W]: Ionophoric action of trans-isohumulone of Lactobacillus brevis. J Gen Microbiol 1993, I39: I04 I- 1045.

2. Sami M, Yamashita H, Hirono T, Kadokura H, Kitamoto K, Yoda K, Yamasaki M: Hop-resistant Lactobacillus brevis contains a novel plasmid harboring a multidrug resistance-like gene. J Ferment Bioeng 1997, 84: I-6.

3. Haakensen M, Schubert A, Ziola B: Multiplex PCR for putative Lactobacillus and Pediococcus beer-spoilage genes and ability of gene presence to predict growth in beer. J Am Soc Brew Chem 2008, 66(2):63-70.

4. Haakensen MC, Butt L, Chaban B, Deneer H, Ziola B, Dowgiert T: A horA-specific real-time PCR for detection of beer-spoilage lactic acid bacteria. J Am Soc Brew Chem 2007, 65(3): I57-I65.

5. Haakensen M, Shubert A, Ziola B: Broth and agar hop-gradient plates used to evaluate the beer-spoilage potential of Lactobacillus and Pediococcus isolates. Int J Food Microbiol 2009, I30(I):56-60.

6. Haakensen M, Pittet V, Morrow K, Schubert A, Ferguson J, Ziola B: Ability of novel ATP-binding cassette multidrug resistance genes to predict growth of Pediococcus isolates in beer. $\mathrm{JAm}$ Soc Brew Chem 2009, 67(3): I70-176.

7. Hayashi N, Ito M, Horiike S, Taguchi H: Molecular cloning of a putative divalent-cation transporter gene as a new genetic marker for the identification of Lactobacillus brevis strains capable of growing in beer. Appl Microbiol Biotechnol 200I, 55(5):596-603.

8. lijima K, Suzuki K, Ozaki K, Yamashita H: horC confers beer-spoilage ability on hop-sensitive Lactobacillus brevis ABBC45cc. J Appl Microbiol 2006, I00(6): I 282.

9. Fujii T, Nakashima K, Hayashi N: Random amplified polymorphic DNA-PCR based cloning of markers to identify the beerspoilage strains of Lactobacillus brevis, Pediococcus damnosus, Lactobacillus collinoides and Lactobacillus coryniformis. J Appl Microbiol 2005, 98(5): I 209-1 220.

10. Klare I, Konstabel C, Werner G, Huys G, Vankerckhoven V, Kahlmeter G, Hildebrandt B, Muller-Bertling S, Witte W, Goossens H: 
Antimicrobial susceptibilities of Lactobacillus, Pediococcus and Lactococcus human isolates and cultures intended for probiotic or nutritional use. J Antimicrob Chemother 2007, 59(5): $900-912$.

II. Klare I, Konstabel C, Muller-Bertling S, Reissbrodt R, Huys G, Vancanneyt M, Swings J, Herman G, Witte W: Evaluation of new broth media for microdilution antibiotic susceptibility testing of lactobacilli, pediococci, lactococci, and bifidobacteria. Appl Environ Microbiol 2005, 7 I ( 2 2):8982-8986.

12. Ammor MS, Belén FA, Mayo B: Antibiotic resistance in non-enterococcal lactic acid bacteria and Bifidobacteria. Food Microbiol 2007, 24(6):559-570.

13. Danielsen M, Simpson PJ, O'Connor EB, Ross RP, Stanton C: Susceptibility of Pediococcus spp. to antimicrobial agents. J Appl Microbiol 2007, 102(2):384-389.

14. Tankovic J, Leclercq R, Duval J: Antimicrobial susceptibility of Pediococcus spp. and genetic basis of macrolide resistance in Pediococcus acidilactici HM3020. Antimicrob Agents Chemother 1993, 37(4):789-792.

15. Billot-Klein D, Gutmann L, Sable S, Guittet E, van Heijenoort J: Modification of peptidoglycan precursors is a common feature of the low-level vancomycin resistant VANB-type Enterococcus D366 and of the naturally glycopeptide-resistant species Lactobacillus casei, Pediococcus pentosaceus, Leuconostoc mesenteroides, and Enterococcus gallinarum. J Bacteriol I994, I 76:2398-2406

Publish with Bio Med Central and every scientist can read your work free of charge

"BioMed Central will be the most significant development for disseminating the results of biomedical research in our lifetime. "

Sir Paul Nurse, Cancer Research UK

Your research papers will be:

- available free of charge to the entire biomedical community

- peer reviewed and published immediately upon acceptance

- cited in PubMed and archived on PubMed Central

- yours - you keep the copyright 